\title{
Supporting Information: Rapid imaging of pulsed terahertz radiation with spatial light modulators and neural networks
}

\author{
Rayko Ivanov Stantchev ${ }^{1, \dagger}, K^{\prime}$ aidi $\mathrm{Li}^{1, \dagger}$, and Emma Pickwell-MacPherson ${ }^{1,2,{ }^{\star}}$ \\ ${ }^{1}$ Chinese University of Hong Kong, Electronic Engineering, Hong Kong SAR, China \\ ${ }^{2}$ University of Warwick, Physics, Coventry, CV4 7AL, United Kingdom \\ *e.macpherson@warwick.ac.uk \\ †These authors contributed equally
}

\section{Contents}

S1 Adaptive sampling distribution in continuous ODU mode

S2 ODU speed and SLM switch rate relationship

S3 Single-pixel imaging

S4 Raw signals and processing

S5 Convolutional neural network

References

\section{S1 Adaptive sampling distribution in contin- uous ODU mode}

The full details of our experimental setup are shown in our previous work of reference ${ }^{1}$, here we focus on the synchronization between the ODU and the THz SLM. As mentioned the main manuscript, the continuous ODU movement mode can in theory achieve a temporal sampling distribution that spends more time where the absolute values of the THz-pulse amplitudes are large. This would be concentrating the measurements around the $\mathrm{THz}$-pulse in a similar way to the adaptive step-mode demonstrated in the main manuscript and in reference $^{2}$. There are two ways in which this can be accomplished; first, with prior knowledge of the pulse amplitudes one can set the acceleration of the ODU accordingly, and second using the voltage from the $\mathrm{THz}$ detector to adjust the acceleration in a feedback loop. The first method would be useful for quality control applications where you know how the temporal THz-trace looks like for a good sample. The second idea does not require any knowledge of the sample, which is its main advantage, however the implementation is more complex. Below we discuss a possible implementation of the second idea.

For the analysis here we will assume that the ODU speed and the voltage to the motor controlling the mirror position are linearly related. For this case we can implement a feedback schema as shown in supplementary figure S1a, the $\mathrm{THz}$ detector voltage readout goes through a low-pass filter, the signal is amplified, then we take the absolute value and there is a negative offset from the signal. Next this corrected voltage gets subtracted from a constant voltage input which is then finally multiplied by 1 or -1 depending on whether the ODU should be moving forward or backwards. The lowpass filter needs to filter out any sudden changes in the $\mathrm{THz}$ signal that would be caused by consecutive masks having very large differences in their read-out values as well as any high-frequency noise. Amplifying the signal is needed in order to get the correct sensitivity of the feedback, for example if the $\mathrm{THz}$ detector voltage output is $20 \mathrm{mV}$ but the ODU requires a $30 \mathrm{~V}$ input then this feedback will result in identical behaviour as having no feedback. Taking the absolute value of the signal is then needed as we do not want the ODU to move faster where the $\mathrm{THz}$ amplitudes are negative as these temporal regions still carry a lot of $\mathrm{THz}$-energy. The next offset value is to make the ODU move faster where there are small THz-amplitudes. A separate loop that decides if the ODU needs to move backwards or forwards. Note, in the event that one uses carrier-wave modulation of the $\mathrm{THz}$ signal, as is done here with further details below and in the supplementary information of reference ${ }^{1}$, then one would need to multiply the $\mathrm{THz}$ signal by this carrier-wave prior to the low-pass filter.

Supplementary figure S1b shows our simulated ODU speed (top) and ODU position (bottom) with and without feedback from the $\mathrm{THz}$ detector as implemented by the schema in part a. The THz pulse used in the simulation is shown in the bottom right corner. It can be seen that ODU moves quicker where there is low $\mathrm{THz}$ intensities and then slows down at the large $\mathrm{THz}$ intensities compared to the no feedback case. The gain and offset values would need to be optimized to achieve the best sensitivity and they might be constrained by the maximum acceleration of the ODU. Note, the offset is not necessary as increasing the constant voltage input would achieve similar behaviour.

Unfortunately, our equipment does not have the capability of implementing anything like the schema presented here. For this reason, we only present this idea and we only focus on the case with no feedback in the below sections. 
a

THz detector feeback into ODU

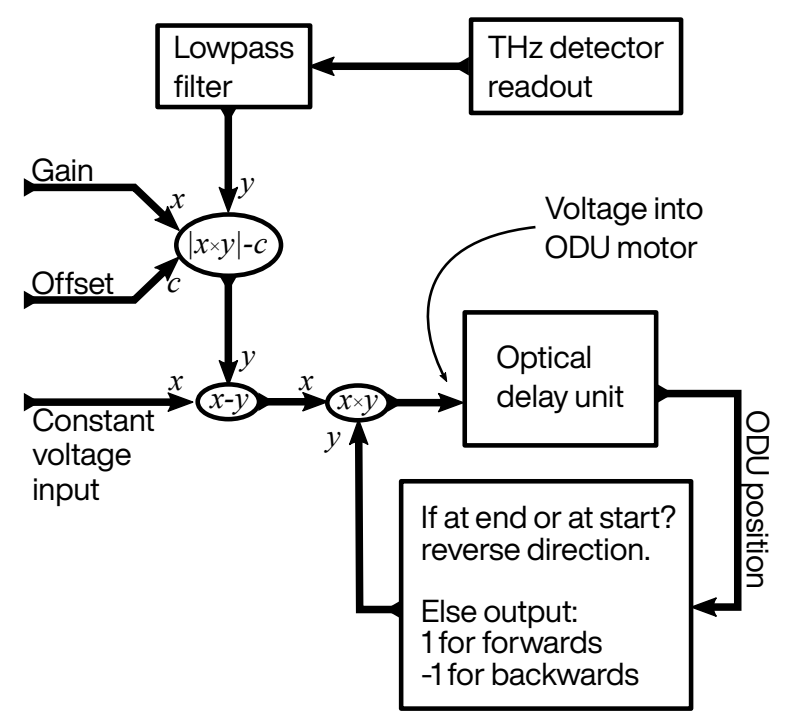

b

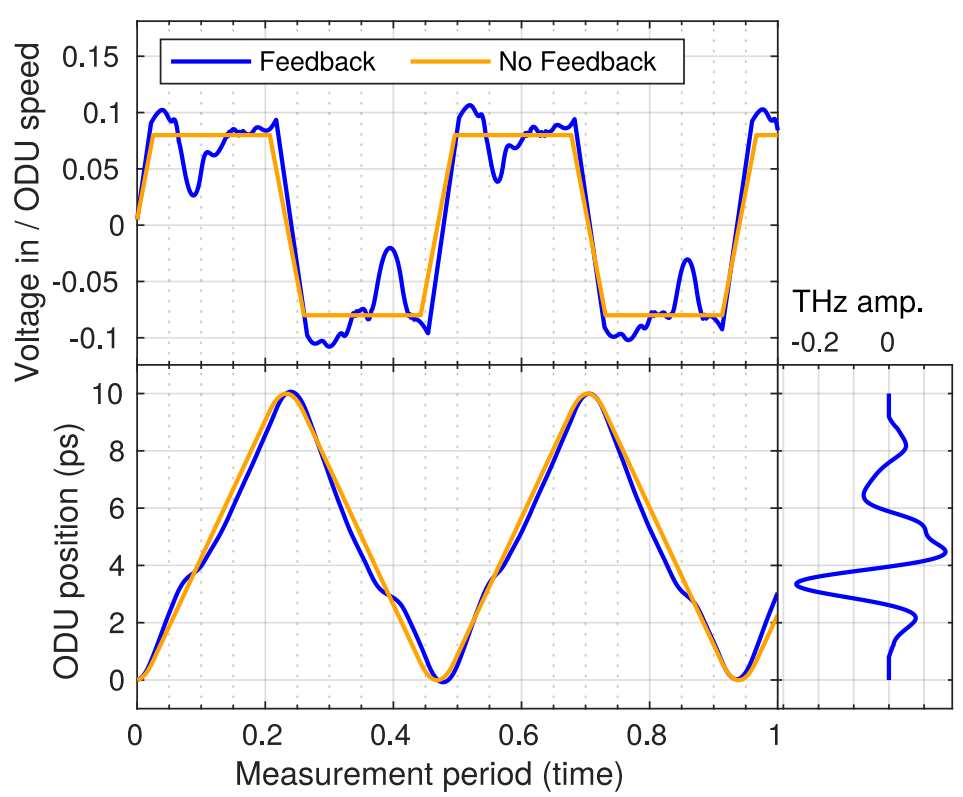

Figure S1. a Scheme for feedback from the THz detector to control the ODU movement speed. b Simulation of the ODU speed with (without) feedback from the THz detector shown by the blue (yellow) line. The ODU position is shown directly below and the $\mathrm{THz}$ pulsed used in simulation is shown on the right.

\section{S2 ODU speed and SLM switch rate rela- tionship}

The relationship of the ODU speed and the SLM switch rate for this system are critically important to the successful implementation of temporal undersampling. As mentioned in the main manuscript, to discuss this idea we will think of the projection of patterns as taking the form of a stepped saw-wave with frequency $f_{s}$ and the ODU position following a triangle-wave motion with frequency $f_{t}$. Further, our hardware does not have a method of keeping the position accurately enough for us to study the case when $f_{t}$ is very large, hence we only study the cases when $f_{s}>f_{t}$. Further, the acceleration and deceleration of the ODU to large speeds would cause the measurements to be more concentrated around the start and end points of the temporal region.

As briefly discussed in the main manuscript, it is critical for $f_{s}$ and $f_{t}$ to not be multiples of each other. The reason for this is that that during the consecutive triangle-wave cycles of the ODU we do not obtain new temporal information but only better averaging for each mask value if they are multiples. This can clearly be seen by looking at a supplementary figure S2, where in part a we plot a triangle-wave with 2 cycles (in blue) and two saw-waves performing 17 and 18 cycles in yellow and red respectively. Below in part $\mathbf{b}$ we plot the scatter plot between the ODU position and the two saw-waves. This scatter plot shows at what temporal point the different masks were sampled. It can be seen that each mask has more temporal sampling-points when the saw-wave performs 17 cycles compared to 18 cycles. For example, $50^{\text {th }}$ mask has 17(9) temporal samplingpoints for yellow (red) saw-wave. This is because 17 is not a multiple of 2 , consequently $f_{s}$ and $f_{t}$ are not multiples of each other, where as 18 is. Thus, when the ODU performs its second cycle the masks are projected at the same temporal-points as when the ODU performed its first cycle.

Supplementary figure S3 shows the sampling scatterplots when the saw-wave and triangle-wave frequencies vary from 1 to 12 in integers. It can be seen that frequencies which obey $m f_{s}=n f_{t}$ for $n, m$ positive integers have the same sampling pattern, for example frequency pairs $\left\{f_{s}, f_{t}\right\}=\{2,1\}$ and $\left\{f_{s}, f_{t}\right\}=\{4,2\}$ result in the same pattern. Therefore, these frequency configurations would end up having the same result, however the averaging method would be different assuming the total measurement period is the same: for $\left\{f_{s}, f_{t}\right\}=\{2,1\}$ we would have measured each mask for twice as long where as for $\left\{f_{s}, f_{t}\right\}=\{4,2\}$ where we have measured each mask twice at the same temporal position. To avoid such redundancy we should choose our frequencies such that they are both prime numbers, or in a more useful way of thinking in terms of implementation is to set the number of cycles of each waveform to be prime numbers during our measurement period. 

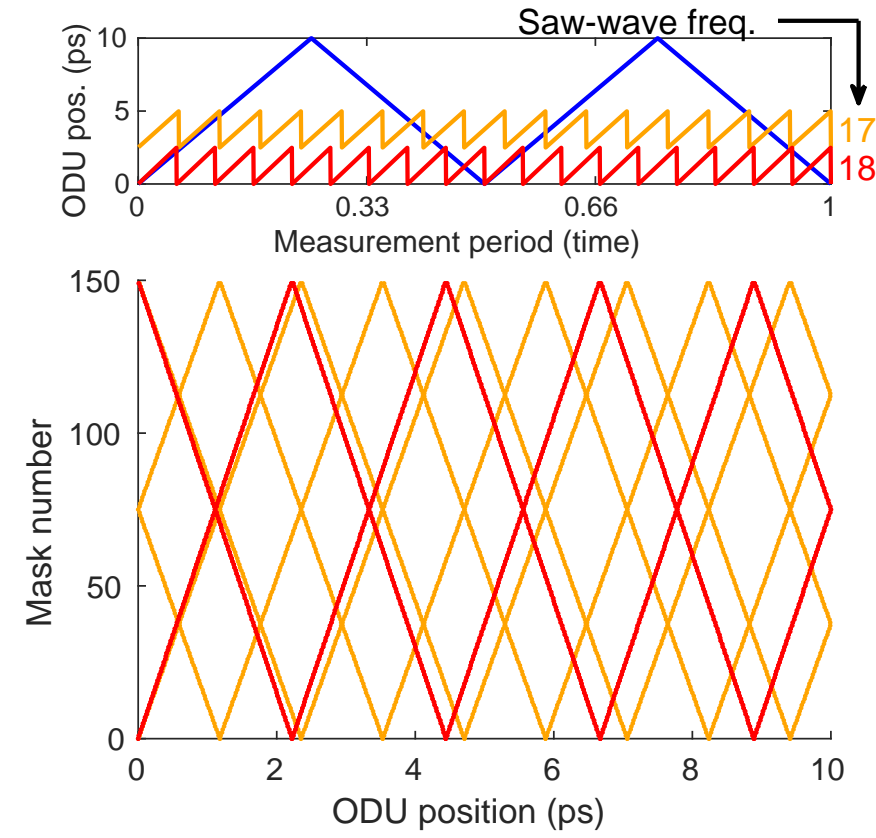

Figure S2. Top: The ODU position shown by the blue line, and the yellow and red saw-waves show the projection pattern of our masks. The yellow (red) saw-wave has performed 17 (18) cycles and the ODU trianglewave has performed 2 cycles during our measurement period. Bottom: Scatter plot of the two saw-wave against the ODU triangle-wave, with horizontal axes showing the ODU position and vertical showing the mask number.

\section{S3 Single-pixel imaging}

\section{D Hadamard single-pixel imaging}

Hadamard single-pixel imaging (HSI) is based upon the Hadamard transform ${ }^{3-5}$, that is HSI obtains an object's Hadamard transform and recovers the image by the inverse Hadamard transform. In detail, the Hadamard spectrum comprises a group of Hadamard coefficients, which correspond to the Hadamard basis pattern in the spatial domain. Each Hadamard coefficient is acquired by a single-pixel detector, which records the dot product of the object and the Hadamard basis pattern projected on it. The two dimensional Hadamard transform $\mathcal{H}\{\}$ can be performed on an $N \times N$ image $I$ via a matrix product:

$$
I_{\mathcal{H}}=\mathcal{H}\{I\}=H I H,
$$

where $H$ is an $N \times N$ Hadamard matrix. However, using the common Hadamard matrices as constructed by Sylvester construction technique will result in a Hadamard transform that is called "natural" or "Hadamard" ordered. This transform will not congregate coefficients with large amplitudes around one region, like the Fourier transform, hence making this

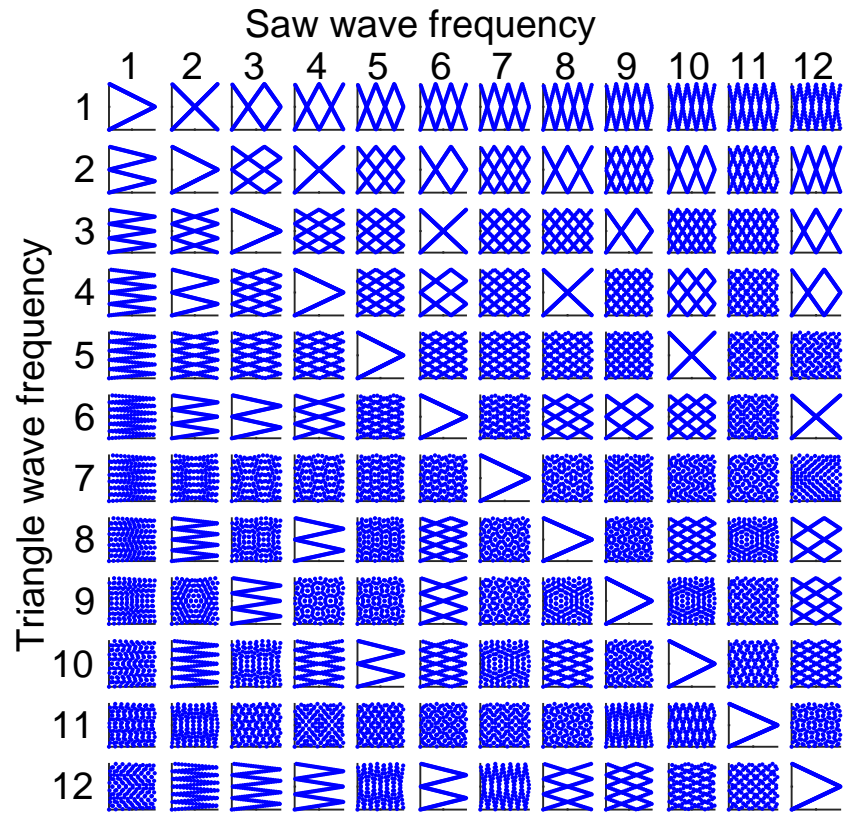

Figure S3. Scatter plots of different saw-waves and triangle-waves when their frequencies vary from 1 to 12 in integers, with the frequency indicated by the vertical and horizontal numbers, ie. the plots in the $3^{\text {rd }}$ column all have a saw-wave with frequency 3 and the plots in $6^{\text {rd }}$ row all have triangle-wave of frequency 6 .

Hadamard transform not suitable for undersampling. We are instead interested in the "sequency" ordered Hadamard transform which arranges things such that coefficients with low sequency come first. The sequency of a Hadamard coefficient is defined as the number of sign changes in its basis pattern, and it is the equivalent of frequency in the Fourier trasnform because the Hadamard transform can be thought of as a decomposition into square waves as opposed to sinusoidal waves. In supplementary figure S4 we show an image and its sequency ordered 2D Hadamard transform. It can be seen that the coefficients with large values are in the top-left corner, which is our $(0,0)$ coordinate. This means most of the information of the image is mainly distributed in the low sequency area meaning that under-sampling can be realized with little reduction in image quality.

In terms of implementation, the most calculation efficient method is to perform the Fast Hadamard Transform along each row and then along each column. Fast Hadamard Transform algorithms are usually based on expressing the coefficients as a series ${ }^{3}$ :

$$
I_{\mathcal{H}}(u, v)=\sum_{x=0}^{N-1} \sum_{y=0}^{N-1} I(x, y)(-1)^{q(x, y, u, v)},
$$

where $(x, y)$ and $(u, v)$ are the coordinates in the spatial 

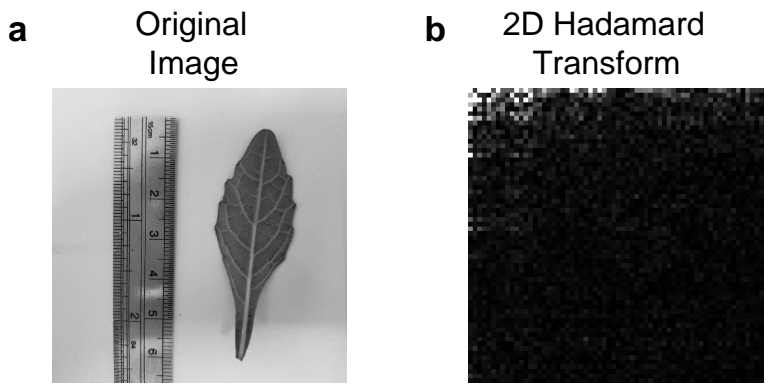

Figure S4. $\mathbf{a}$ The original image and $\mathbf{b}$ shows its corresponding 2D Hadamard transform with sequency ordering.

and Hadamard domain respectively, and

$$
q(x, y, u, v) \equiv \sum_{i=0}^{n-1}\left(g_{i}(u) x_{i}+g_{i}(v) y_{i}\right),
$$

where $n=\log _{2}(N)$ and $g_{i}(u)$ being defined as

$$
\begin{aligned}
g_{0}(u) & \equiv u_{n-1} \\
g_{1}(u) & \equiv u_{n-1}+u_{n_{2}} \\
g_{2}(u) & \equiv u_{n-2}+u_{n-3} \\
& \vdots \\
g_{n-1}(u) & \equiv u_{1}+u_{0} .
\end{aligned}
$$

The symbols $u_{i}, v_{i}, x_{i}$ and $y_{i}$ are the bits of the binary representations of $u, v, x$ and $y$, ie.

$$
(u)_{\text {decimal }}=\left(u_{n-1} u_{n-2} \ldots u_{1} u_{0}\right)_{\text {binary }} \text {. }
$$

Supplementary equation S4 determines the ordering of the Hadamard transform, and this is the definition for the sequency ordering we are interested in.

To acquire the Hadamard coefficient through the single-pixel detector, Hadamard basis patterns need to be generated and projected onto the object. A Hadamard mask pattern can be generated by:

$$
P_{\mathcal{H}}^{u_{0}, v_{0}}(x, y)=\mathcal{H}^{-1}\left\{\delta\left(u-u_{0}, v-v_{0}\right)\right\},
$$

where $\delta(u, v)$ is the delta function. In supplementary figure $\mathrm{S} 5$ we show Hadamard basis masks by substituting $\left(u_{0}, v_{0}\right)$ with $(1,1),(1,3),(1,9),(4,1)$ is part a to $\mathbf{d}$ respectively. As can be seen from part $\mathbf{a}$, the position in the Hadamard spectrum is $(1,1)$ hence we have one sign change along each row and one sign change along each column.

Note that our SLM can only project [1 0 ] masks. However we can perform a differential measurement, which in effect allows us to measure each Hadamard coefficient $I_{\mathcal{H}}(u, v)$ as if we had used [1 -1] masks ${ }^{6-8}$. However, this needs two measurements to get one coefficient; the first measurement is acquired through projecting the Hadamard basis pattern on the object and
2D Hadamard masks

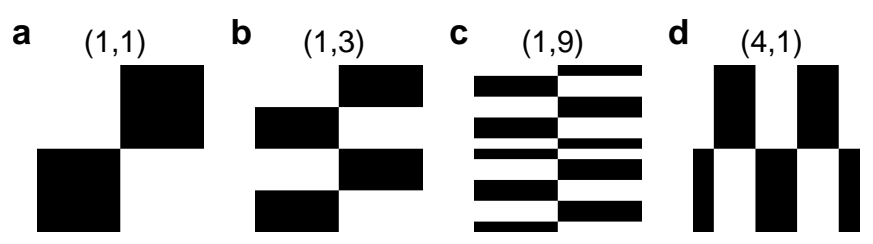

Figure S5. The generated Hadamard basis masks. The first number on top of each figure shows the sequency in the row and the second one shows the sequency in the column. The number pair denotes the corresponding Hadamard coefficient position in the Hadamard domain.

the second measurement is acquired by projecting its inverse. The second measurement is then subtracted from the first, giving the signal that would be measured if we had projected masks with [1 -1] values. This process takes two times the number of Hadamard coefficients but it has better suppression of noise. All the Hadamard coefficients are real-valued and the number of coefficients equals the number of image pixels if we fully sample it.

\section{Fourier single-pixel imaging}

Two-dimensional images can be represented by a combination of sinusoidal patterns. This the idea of performing the 2D Fourier transform $\mathcal{F}\{\}$ on an image $I(x, y)$ which is a defined as:

$$
\begin{aligned}
I_{\mathcal{F}}(u, v) & =\mathcal{F}\{I(x, y)\} \\
& =\sum_{x=0}^{M-1} \sum_{y=0}^{N-1} I(x, y) e^{-2 \pi i\left(\frac{u x}{M}+\frac{v y}{N}\right)},
\end{aligned}
$$

where $x, y$ are the spatial co-ordinates with $u, v$ being Fourier domain co-ordinates. In the context of singlepixel imaging, the masks that we project are obtained by the inverse Fourier transform $\mathcal{F}^{-1}\{\}$ of delta functions at different positions;

$$
P_{\phi}(x, y)=\frac{1}{2} \text { real }\left(\mathcal{F}^{-1}\left\{\delta\left(u-u_{0}, v-v_{0}\right) e^{i \phi}\right\}+1\right),
$$

where $\delta(u, v)$ is the delta function. Note, the +1 and the $1 / 2$ come from the fact that our masks have values between 0 and 1. Projecting these illumination patterns $P_{\phi}(x, y)$ on an object and measuring all the light transmitted (or reflected) gives us a measurement we call

$$
D_{\phi}^{u_{0}, v_{0}}=\sum_{x=0}^{M-1} \sum_{y=0}^{N-1} I(x, y) P_{\phi}(x, y) .
$$

The goal is then to project a set of patterns and obtain a set of measurements from which we can reconstruct our image. Since our projected masks can only 
have values between 0 and 1, we will have to perform a subtraction of different measurements to obtain the equivalent of a mask having values between 1 and 1 , as is necessitated by the Fourier transform. Since each Fourier coefficient can be represented by a cosine and sine wave, we can acquire each complex-valued Fourier coefficient $I_{\mathcal{F}}(u, v)$, by projecting three illumination patterns $\left\{P_{0}(x, y), P_{2 \pi / 3}(x, y), P_{4 \pi / 3}(x, y)\right\}$ and using the corresponding three measurements via the relation

$$
\begin{aligned}
\mathcal{F}\left(u_{0}, v_{0}\right)= & 2 D_{0}^{u_{0}, v_{0}}-D_{2 \pi / 3}^{u_{0}, v_{0}}-D_{4 \pi / 3}^{u_{0}, v_{0}} \\
& +\sqrt{3} i\left(D_{2 \pi / 3}^{u_{0}, v_{0}}-D_{4 \pi / 3}^{u_{0}, v_{0}}\right) .
\end{aligned}
$$

This is repeated until we have collected the Fourier spectrum of the object with its image obtained by the inverse Fourier transform. Here, we used a three shift method to acquire each complex-value Fourier coefficient because the four step method requires more measurements. Further, the prior knowledge that the image is real-valued results in a Fourier spectrum that is conjugate symmetric, therefore fully sampling an $M \times N$ pixel image using three shift method takes $3 / 2 \times M \times N$ measurements since $\mathcal{F}\left(-u_{1},-v_{1}\right)=\mathcal{F}^{*}\left(u_{1}, v_{1}\right)$ where * denotes the conjugate.

\section{D Fourier masks}

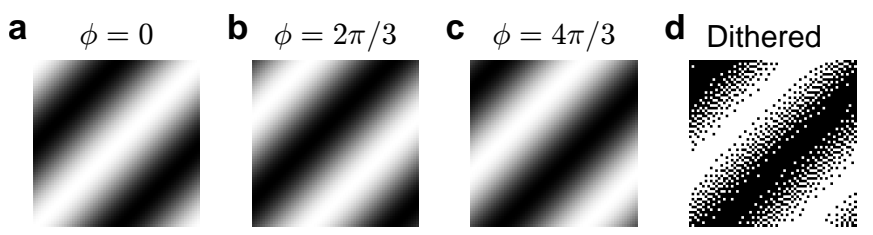

Figure S6. a-c Fourier masks for the same Fourier coefficient however with different phases. d The dithered mask version of part $\mathbf{c}$.

\section{Grayscale valued masks}

Obtaining an image with a spatial light modulator (SLM) works by projecting a set of masking patterns onto an object and using a single-element detector to record the resulting transmission (or reflection) of an object for each pattern. Our THz SLM works by patterning a visible light beam with a digital micromirror device (DMD) and shining these masking patterns on to a piece of silicon, where the photoexcitation of charge carriers increases the silicon conductivity thus decreasing the $\mathrm{THz}$ transmission (or reflection). The masking patterns incident on the silicon are determined by the the DMD mirror states which is either on or off, i.e. binary masks. Obtaining grayscale modulation values with such a system is possible mainly due to difference of sizes of the DMD mirrors and carrier diffusion lengths.
The charge carrier mobility is defined as the magnitude of its drift velocity per unit electric field i.e. $\mu=$ $\left|V_{a v g}\right| / E$, where $V_{\text {avg }}$ is the electron drift velocity and $E$ is the electric field. The generated conduction electrons and holes will diffuse according to the 3D diffusion equation, hence their mean square diffusion length is:

$$
<x^{2}>=6 D t
$$

Where $D$ is the diffusion coefficient given by the Einstein-Smoluchowski relation $D=\mu_{q} k_{B} T / q=$ $\tau_{c} k_{B} T / m_{e}$ for carrier mobility $\mu_{q}=q \tau_{c} / m_{e}, \tau_{c}$ is carrier scattering time and $m_{e}$ carrier effective mass. In supplementary equation $\mathrm{S} 11 t$ is the carrier life time which was measured to be $65 \mu$ s for our silicon wafer, thus the calculated diffusion length is around $1 \mathrm{~mm}$.

The size of the individual DMD mirrors of $10 \times 10 \mu \mathrm{m}$ is very small compared to the carrier diffusion length, noting that we project our DMD with a magnification of 1. This size difference allows us to obtain grayscale modulation values as follows. Consider turning on two adjacent mirrors; the projected pattern will look identical to turning on just one mirror however the the total number of charge carriers in this region will be doubled. Therefore, if we binarize our Fourier mask by spatial dithering, as shown in supplementary figure S6d, then the diffusion of charge carriers will blur this binarized image and create a grayscale absorption profile for the $\mathrm{THz}$ beam. However, the $\mathrm{THz}$ absorption and number of charge carriers do not have a linear relationship as shown in supplementary figure S7, therefore we need to perform a calibration step between the actual modulated value and projected grayscale value accordingly.

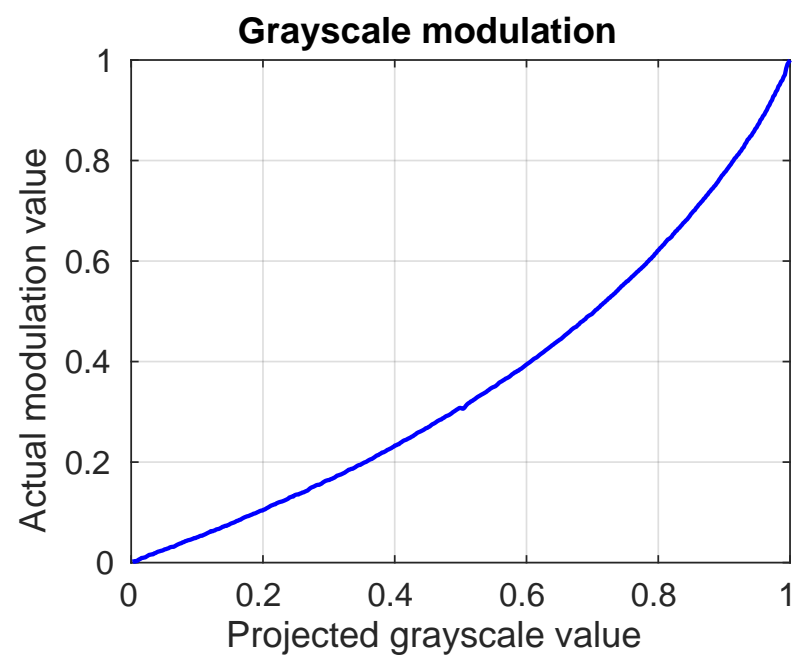

Figure S7. The relationship between the actual modulation value and projected grayscale value. 


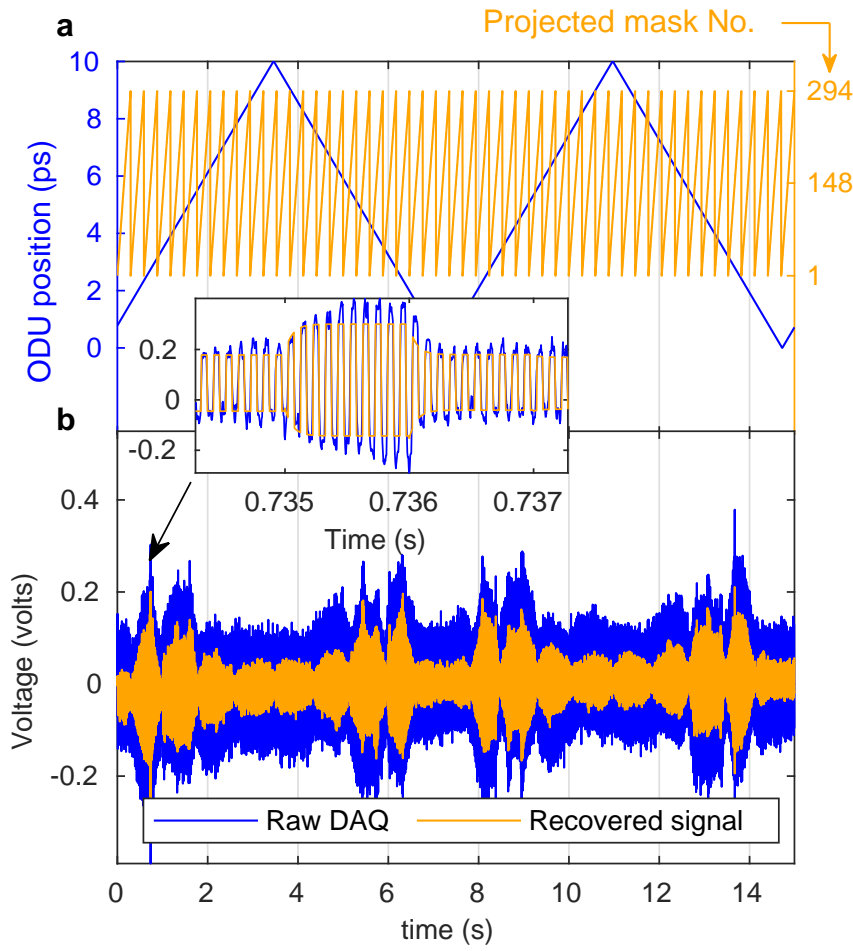

Figure S8. a The ODU position (blue) and the projected masks (yellow) during our measurement period of 15 seconds. b The raw (recovered) signals shown as the blue (yellow) line. The inset shows a close up of signals over a duration of $4 \mathrm{~ms}$. Note, we used 294 Fourier masks with a switch rate of $1 \mathrm{kHz}$ for this example.

\section{S4 Raw signals and processing}

In supplementary figure S8 we shows an example of the raw signals from our measurement. Part a shows our ODU position in blue and the projected masks in yellow. The horizontal axis is time with out measurement period being 15 seconds for the example shown here. Part b shows raw $\mathrm{THz}$ signal in blue and recovered signal in yellow. How we obtain the recovered signal is by saying that each time segment for each projected mask has an amplitude and we find the amplitude that best fits our data, with the full mathematical details shown in our previous work of reference ${ }^{1}$. The inset shows a close up of our signals over a period where we have projected mask three different masks. It can be seen that the signals have larger amplitudes when the ODU is somewhere around 2 and 4 ps with the signals having a near constant amplitude when the ODU is between 5 and $10 \mathrm{ps}$. This is because our THz-pulse is centered around 3 ps. Another feature that can be seen is some spikes in the raw signal. Some of these are due to a mask having a large amplitude compared to the mask value around it and others are just electrical noise.

After processing and extracting the amplitudes for each projected mask, we obtain supplementary figure
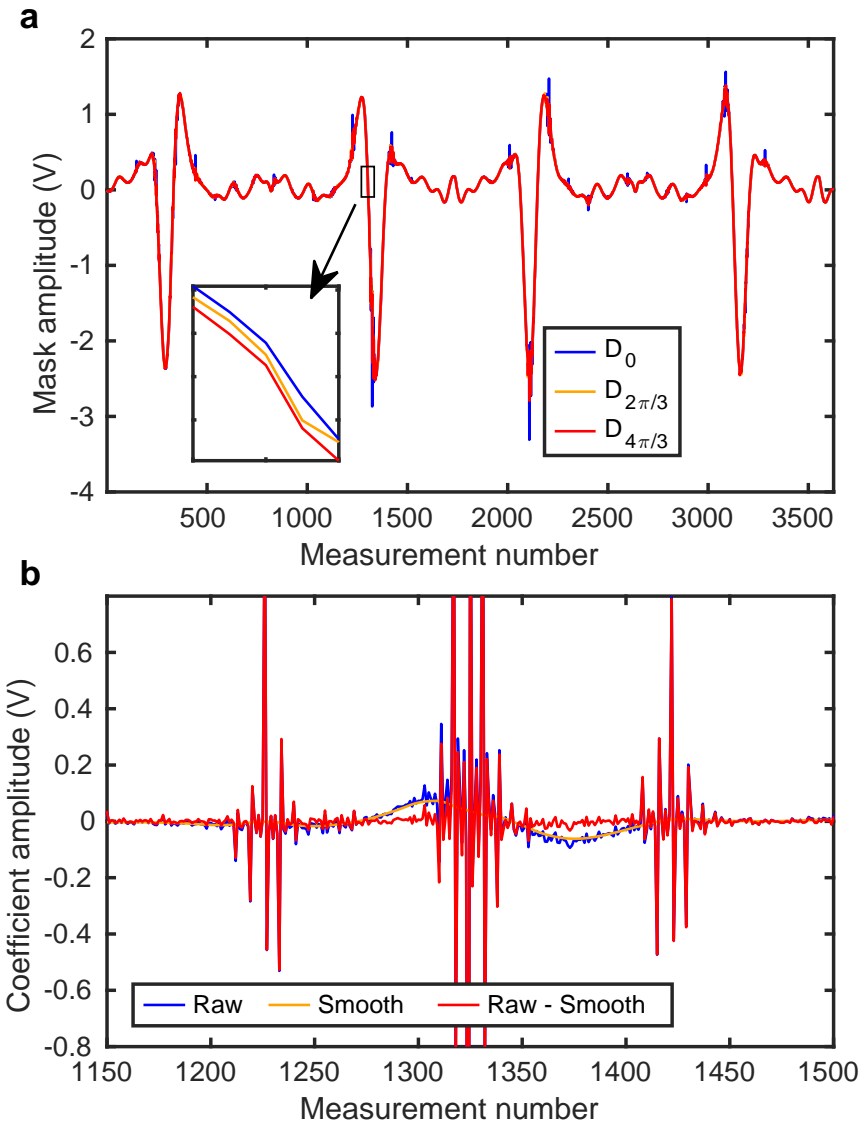

Figure S9. a Extracted amplitudes of the Fourier masks as a function of measurement number. The masks from the different phases $\phi=\{0,2 \pi / 3,4 \pi / 3\}$ have been separated as the blue, yellow, red lines respectively. The inset shows a close up of the masks amplitudes where the $\mathrm{THz}$ pulse has very steep gradient. $\mathbf{b}$ The blue line shows the raw unprocessed coefficient value, the real part, as obtained by supplementary equation $\mathrm{S} 10$. The smooth signal (yellow line) is obtained by taking moving average of the $\left\{D_{0}, D_{2 \pi / 3}, D_{4 \pi / 3}\right\}$ signals above, applying the same equation, and then taking the moving average again. Red line is the raw subtract the smooth signal.

S9a. For the example shown here we use Fourier masks derived from the 3-step method as discussed in supplementary section S3, however the analysis for using Hadamard derived masks is identical. Here the $\mathrm{THz}$ pulse shape becomes much more obvious. Some spikes in the blue line, resulting from the $D_{0}$ masks, can be seen. This is because the first $D_{0}$ mask from the $(1,1)$ delta function, ie. the $0^{\text {th }}$ frequency, has the largest amplitude since it consists of all 1s (transmits everything). Equation S10 shows how to obtain the relevant Fourier coefficient from the measurements in part $\mathbf{a}$ with the resulting values in show in part $\mathbf{b}$. A low frequency oscillation can be seen in the raw (blue) line 
around the coefficients with large amplitudes. These oscillations are artefacts from our measurement; they arise from the fact that although we display each consecutive mask one after the other then perform subtraction to obtain the coefficient value, the masks were not taken at the same temporal point. This creates subtle amplitude value differences where $\mathrm{THz}$ pulse gradients are large, as can be seen in the inset of supplementary figure S9a. These oscillatory artefacts can be removed by subtracting the smooth signal show by the yellow line. The smooth signal is obtained by taking the moving mean of the individual $D_{0}, D_{2 \pi / 3}, D_{4 \pi / 3}$ signals, then applying equation S10 to these new signals and finally taking the moving mean again. Then subtracting this smooth signal from the raw one we obtain red curve in supplementary figure S9b. Note that these artefacts are much more subtle than the ones described in main manuscript and not removing them, as described here, will not significantly degrade image quality. These artefacts are about 30 times smaller than the those caused by only using [ $\left[\begin{array}{ll}1 & 0\end{array}\right]$ masks.

\section{D image reconstruction}

Next we discuss our method of constructing a 3D image from the data presented in supplementary figures S8 and S9. For this example we use 98 Fourier coefficients as the measurements. Since we know the position of the delay line when every measurement was taken as well as the mask we were projecting at that time, we can create a scatter plot of supplementary figure S10a that shows show the value of a coefficient as well as the temporal points at which it was measured. As mentioned in the main text, we are now faced with the problem that different coefficients are sampled at different temporal points, so how do we construct an image at a single temporal point. We have found the best solution to be do linear interpolation so that are all the coefficients are aligned to the same temporal points. For example, if coefficient 30 and 45 were respectively sampled at $\{1.1,2.1,3.4,5.1\}$ and $\{1.2,2.4,3.4,5.0\}$ ps then we can interpolate and extract their values at $\{1.0,2.25,3.5,4.75\}$ ps. This is the step taken to obtain b from part a in supplementary figure S10, where our interpolation step is $0.05 \mathrm{ps}$. Further note, after interpolation we perform a frequency filter that removes any features we do not expect in our data, ie. our THz system can measure up-to $1.7 \mathrm{THz}$ hence we remove anything above this frequency. Finally, we can reconstruct our 3D image by creating a set of 2D images and stacking them together. Note, we tried other techniques for our $3 \mathrm{D}$ reconstruction, such as putting measurements in temporal bins, using the nearest temporal point and non-uniform Fourier transform, however they all had significantly worse performance than linear interpolation.
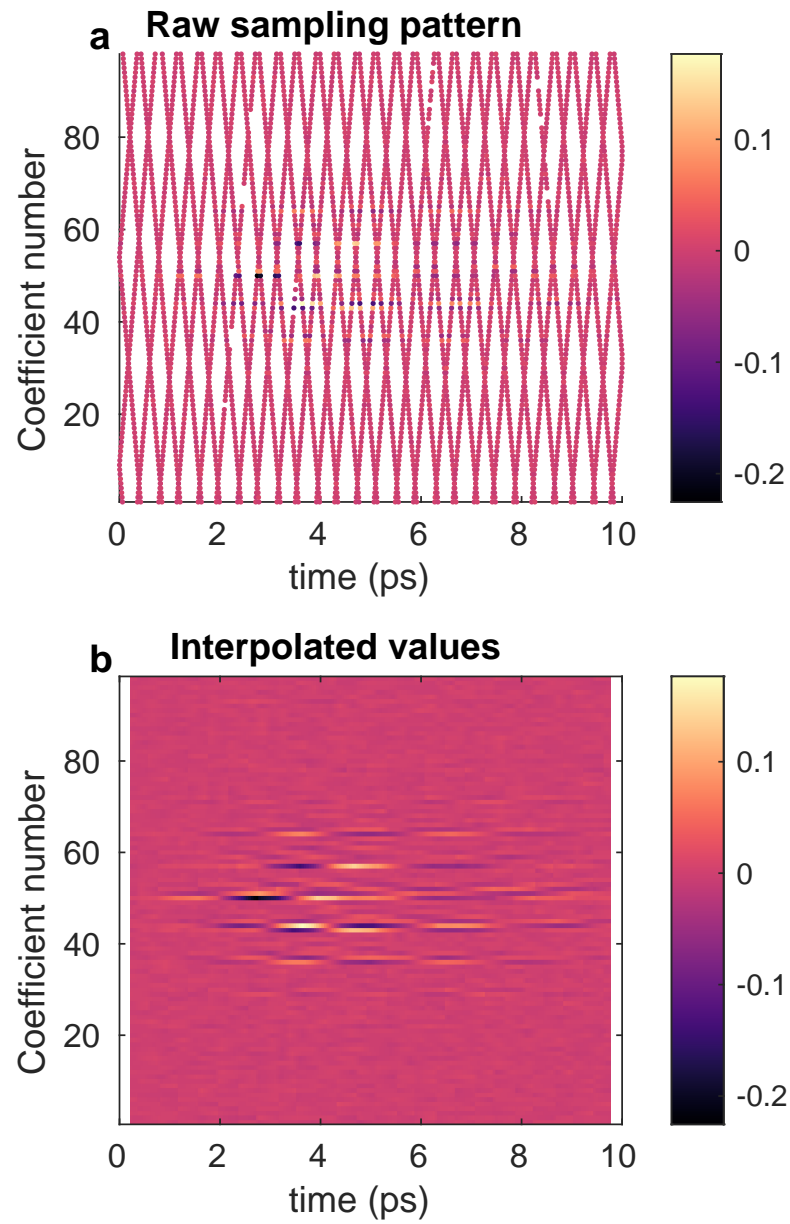

Figure S10. a Scatter plot of the real part of the coefficient amplitudes against the ODU position. b Coefficient amplitudes interpolated at every $0.05 p s$. Note we interpolate along time only, ie. we perform the $1 \mathrm{D}$ interpolation of each coefficient separately. The signals have also been passed through a low-pass filter set at $1.7 \mathrm{THz}$.

\section{S5 Convolutional neural network}

There has been a large interest in applying deep learning (DL) methods for $\mathrm{THz}$ imaging analysis ${ }^{9}$ and enhancement ${ }^{10}$. DL methods have the ability to extract features from a large dataset for further analysis and processing. These techniques have been employed successfully in many aspects such as image denoising $^{11}$, object classification ${ }^{12}$, identification ${ }^{13}$ and phaseimaging ${ }^{14}$. Specifically, DL methods applied in our study show the potential to improve the image quality for single-pixel imaging ${ }^{10}$. It has been observed that images reconstructed from a single-pixel detector contain blurring artifacts and noise. To remove these artifacts and recover high-quality images, which can further improve the single pixel imaging speed, a symmetric deep convolutional neural network (SDCNN) is explored and 
built which learns an end-to-end mapping between the ground truth and under-sampled $\mathrm{THz}$ images. Here we build and design a SDCNN to enhance the $\mathrm{THz}$ image quality recovered from single-pixel imaging, where our data-set contains 38950 two-dimensional $\mathrm{THz}$ images as the training data and $61802 \mathrm{D}$ images act as the test data. The experimental results show that our proposed SDCNN combined with continuous mode sampling in single-pixel imaging can be used to obtain high quality 3D THz images with quick acquisition. This work can provide guidelines for future applications of $\mathrm{DL}$ in $\mathrm{THz}$ technologies.

The proposed SDCNN structure is shown in supplementary fig S11. The network starts by implementing a convolutional layer (Conv2D) with 32 trainable filters to extract $\mathrm{THz}$ image features with a relative large receptive field $(5 \times 5)$. The following Conv2D layer has 64 filters with a smaller receptive field $(3 \times 3)$, which benefits the training with higher efficiency and catches deeper information hidden in the $\mathrm{THz}$ images. In the middle of the network, there is a single Conv2D layer with 128 filters which are of size $3 \times 3$. The second half stage has the Conv2D layer with 64 filters and 32 filters, respectively. The size of the filters in both layers is 3 . The output is recovered by using a single Conv2D filter of size $1 \times 1$. The parameters in the network are initialized by the Xavier initialization which is a popular method in CNN model because of its good capabilities to optimize the loss function ${ }^{15}$. To speed up the training process, each Conv2D layer is followed by a batch normalization (BN) layer ${ }^{16}$. The rectified linear unit(ReLu) nonlinear activation function is performed behind every $\mathrm{BN}$ layer because it has been extensively verified for the excellent performance on avoiding the gradient vanishing problem during the training. The max pooling layers are attached to decrease image dimensions and improve robustness to noise. On the contrary, the upsampling layers improve the image size using the bilinear interpolation in the second half. In the training process, when the data flow through the network pipeline, the max pooling and convolutional operations improve noise-robustness at the expense of losing information about the minor details. To better recover the images, we implement skip connections to pass information directly from the first half to the second half part which saves important details and information and propagate gradients to the deeper layers. The network is elaborately designed and fine-tuned to improve $\mathrm{THz}$ image quality, which would further improve the speed of the single-pixel imaging. Once the network is well trained, the calculation of the test images set is done with ignorable computational time. Note, that after recovering the under-sampled $\mathrm{THz}$ images by single-pixel imaging, the images are fed in the SDCNN for quality enhancement.

To train the network, we need a suitable loss function that improves the image quality without removing real features. Assuming $\mathcal{O}(x, y)$ is the true image of our object, then the recovered image by single pixel imaging using under-sampled measurement is a degraded version, which can be written as:

$$
\mathcal{O}_{\text {meas }}(x, y)=D(\mathcal{O}(x, y))+n
$$

where $\mathcal{O}_{\text {meas }}$ is our measured image, $D$ stands of stands for the degradation process, and $n$ is the noise. Here, our SDCNN is designed to solve the ill-posed inverse problem by estimating the original images from the measured images. To realize this, the network is trained to establish an end-to-end mapping from $\mathcal{O}_{\text {meas }}$ to $\mathcal{O}(x, y)$. To approximate $\mathcal{O}(x, y)$, we obtain a ground truth $\mathcal{O}_{g}(x, y)$ by measuring the same object multiple times using a slower SLM switch rate $(1 \mathrm{~K}$ switch rate, 13 averages) and taking the average. The next step is the design of the loss function which directly decides the objective of the training and the quality of the model. The loss function we designed for the training process is:

$$
\text { Loss }=(1-r) \times \operatorname{SSIM}\left(\mathcal{O}_{c}(x, y), \mathcal{O}_{g}(x, y)\right)+r \times b|\Gamma|,
$$

with $\Gamma$ being a ratio used to preserve the energy in each 2D-image defined as,

$$
\Gamma=\frac{m^{-2} \sum_{x, y=1}^{m} \mathcal{O}_{g}(x, y)+b}{m^{-2} \sum_{x, y=1}^{m} \mathcal{O}_{c}(x, y)+b}
$$

where $\mathcal{O}_{c}(x, y)$ is the output of the network, SSIM is the structural similarity index measure ${ }^{17}, m$ is the length of the image (we interpolate the inputs, ground truth and output images to $96 \times 96$ ), $r$ is an parameter with the range from 0 to 1 used to balance the contribution between the SSIM value and the energy preservation factor $\Gamma$. The constant $b$ is set to 15 to avoid the denominator of the $\Gamma$ being 0 , as all of our ground truths never have a mean value lower than -1 . As the $2 \mathrm{D}$ images are collected at different positions on the time-domain pulse, the average value of each output 2D image corresponds to the pulse value at the temporal point should we be performing $\mathrm{THz}$ spectroscopy instead of imaging. The structural similarity index measure $(\mathrm{SSIM})^{17}$ is a function commonly used to evaluate the similarity between a ground truth and an image that has undergone some degradation process. The SSIM varies between 1 and 0 with 1 when the image is compared to itself.

The network is fed the under-sampled 2D images and corresponding ground truth as recovered from the single pixel imaging. To update the network parameters and minimize the loss function, Adam optimizer is used with back propagation. At the start the learning rate is 


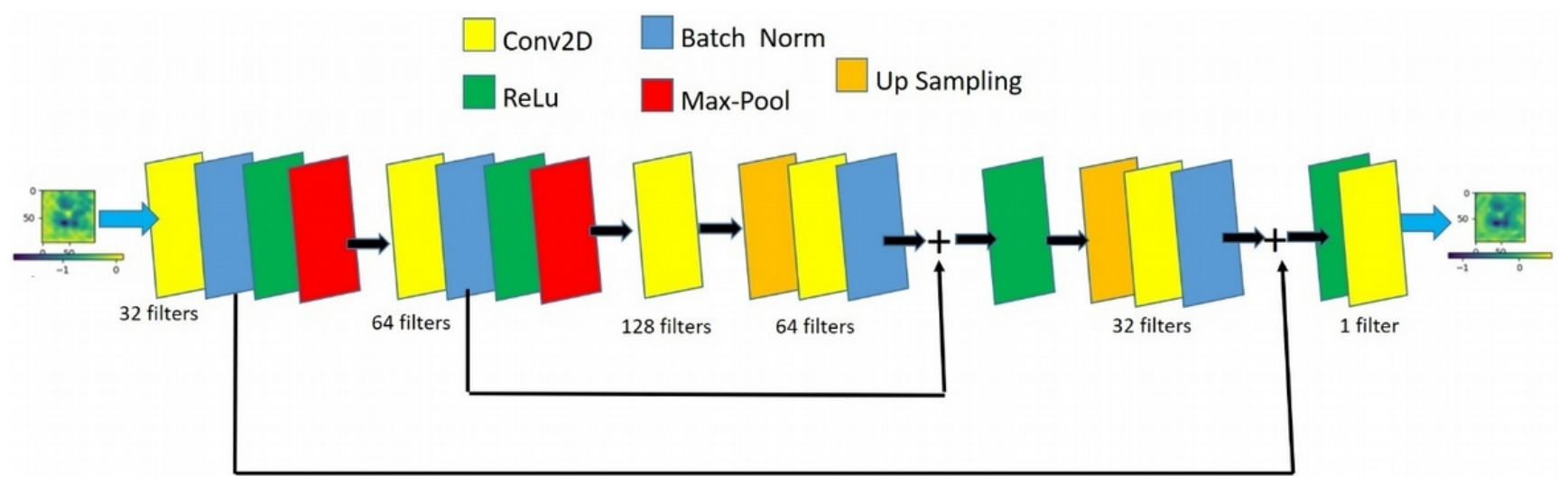

Figure S11. The structure of the symmetric deep convolutional neural network(SDCNN).

set at $10^{-4}$ and after every 10 epochs ( 1 epoch means all the training data has been trained once) of training it will decrease by dividing though by 5 . For the training data, we imaged 15 different objects and another object was as test data for validation purposes. Further, each object was imaged at different temporal undersampling ratios for both step-mode and continuous-mode imaging. Each object had a corresponding ground truth $3 D$ image made up from 205 single 2D-images each from a different temporal position. In the end, the full dataset contains 38950 2D THz image pairs of $96 \times 96$ size which were interpolated from the original $32 \times 32$ sized 2D images. The test image set contains $61802 \mathrm{D}$ image pairs recovered from the step-mode and continuousmode sampling regimes for the same object (two plastic lines shape), which is the individual 3D images at the different temporal sampling ratios studied in the main manuscript. Note, all of this validation test data compared to the ground truth, at each individual temporal point, can be seen in supplementary video 1 along with the outputs from the network. Pytorch packages in PYTHON 3.7 were used to construct the network which can then be trained on CPU or GPU. Note, the network training lasted 3.5 hours on an Nvidia GeForce GTX 980 Ti graphics card whereas on an Intel i7-5820k CPU @3.30GHz training lasted 7 hours.

To evaluate the energy preservation, we introduce the Mean Square Error(MSE) which is defined as:

$$
\mathrm{MSE}=\frac{1}{T m^{2}} \sum_{t=1}^{T} \sum_{x, y=1}^{m}\left(\mathcal{O}_{g}(x, y, t)-\mathcal{O}_{c}(x, y, t)\right)^{2},
$$

where we have $T$ number of temporal points in a single 3D THz image. The smaller value of MSE means that average values of the two images are more similar, thus the better energy preservation and reduction of noise. Supplementary figure S12 shows the relation between MSE and the $r$ value. As can be seen here, the selection of $r$ value is of great importance to the MSE

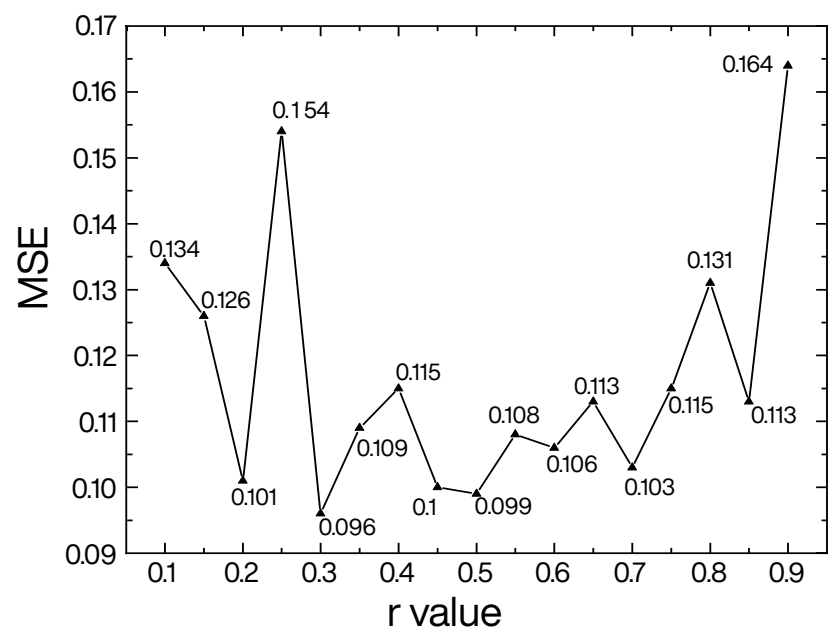

Figure S12. The average MSE value for the test data sampled in continuous mode with different $r$ value in the loss function.

value and therefore the image quality. When $r$ equals to 0.3 the MSE reaches the minimum of 0.096, and then it creases increases with some fluctuations. Note that whilst using the MSE to evaluate the performance of our network might imply that we should have used the MSE in loss function, this is not true as the best function would depend on the application in mind. For example, if one is interested in the $\mathrm{THz}$ frequency spectra, then the loss function should look at the Fourier transformed data and not the time-domain as we have here. We include the MSE for completeness.

Recovered pulse shapes for the test data with different $r$ values at the temporal sampling rate of $73 \%$ in continuous mode are shown in supplementary figure S13. When $r=0.3$, the recovered pulse is closest to the ground truth. While for $r=0.1$ or 0.9 , the pulses are distorted and they also they have higher MSE value. Pulses recovered under other temporal sampling rates show the same trend. 


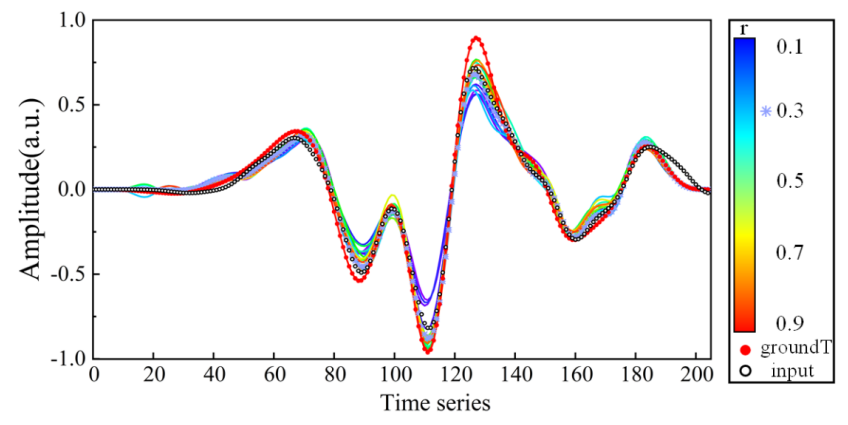

Figure S13. Recovered pulses for the test data in continuous mode with $73 \%$ sampling rate and different $r$ value in the loss function.

Supplementary figure S14 shows the SSIM value of the $3 \mathrm{D} \mathrm{THz}$ test data sets as outputted by the network as a function of temporal sampling ratio for different $r$ values in the loss function. It is obvious that when $r=0.3$ the improvement of the SSIM value is largest whatever the sampling ratio is. Therefore, $r=0.3$ is considered to be the best value to optimize the images by having the best balance between SSIM improvement and energy preservation. The loss value trend in the process of training is shown in $\mathrm{S} 15$ and as expected the loss value keeps decreasing during the training, showing our training is heading a good direction with high efficiency. After 45 epochs, the loss value becomes near saturated at around -0.39 , marking the end of the training process.

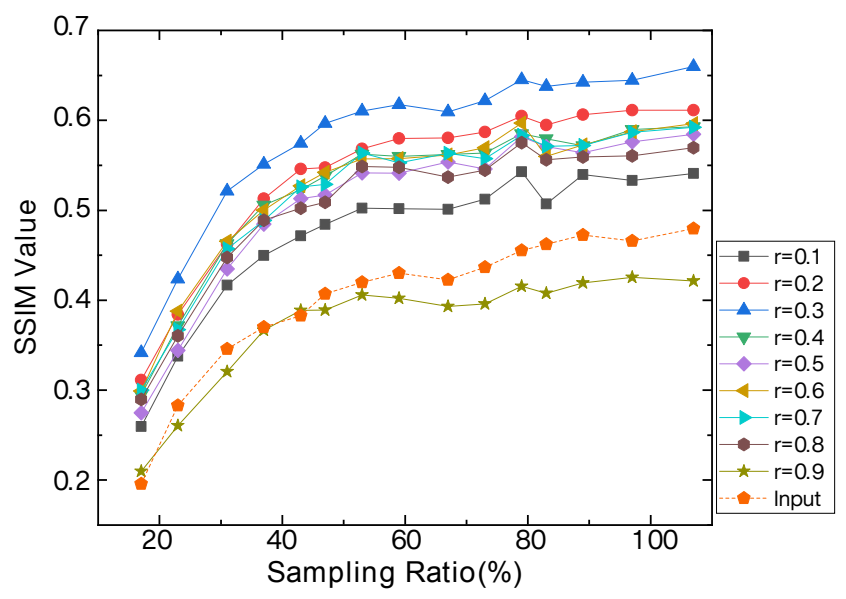

Figure S14. The SSIM value for the outputs of the test data in continuous mode with different sampling ratio under different value in the loss function.

The neural network we built is primarily used to enhance the quality of our images. When we apply our network to an image acquired where our SLM changes masks at a $5 \mathrm{kHz}$ switch rate, we see the SSIM improve from 0.4 to about 0.6 as shown in the main manuscript.

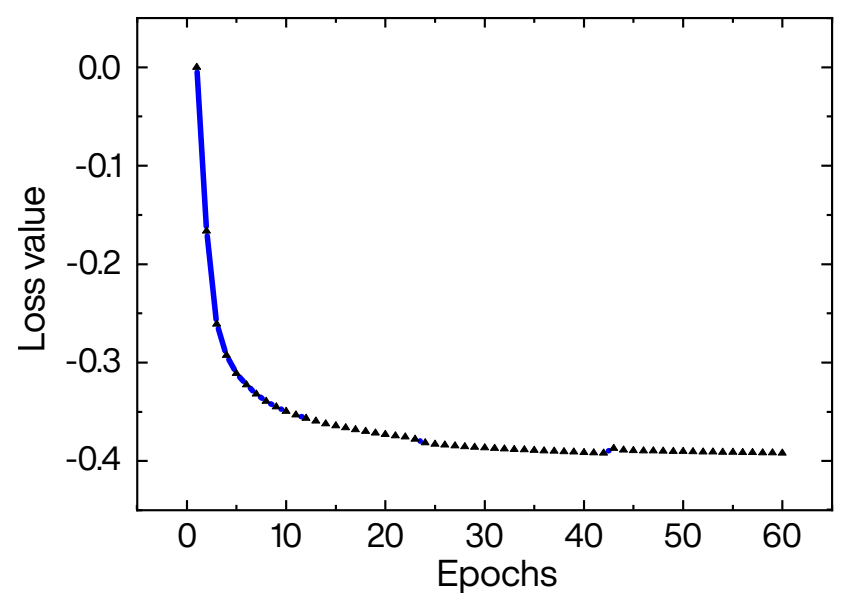

Figure S15. The loss value in the training process.

For comparison, if we set our SLM to change masks at a $1 \mathrm{kHz}$ rate, we acquire images with an SSIM of around 0.6 , as shown in supplementary figure S16. Here we can see that neural network improves the SSIM and retains the same behaviour as the temporal undersampling ratio is reduced. This is because the network was been trained to look at 2D images where as the artefacts from the temporal undersampling can only be seen when looking at images as 3D data set. Training the network to look at the data as 3D images is possible, however training a network to eradicate artefacts at multiple undersampling ratios is inefficient. It would be much more efficient to train a network to eradicate the artefacts at a single undersampling ratio. However, this work concerns itself with showing that an SLM and an ODU can be used together for full time-domain $\mathrm{THz}$ imaging, in particular showing the superiority of the continuous sampling mode and the problems associated with performing temporal undersampling.

\section{Comparison with other image denoising techniques} Using a neural network, however, is not the only way to remove artefacts and noise in our images resulting from the measurement. There other techniques commonly used such as wavelet denoising and total variation minimization algorithms that are commonly used to denoise images. Therefore we compare our results from the neural network to other image denoising techniques, namely total variational minimization (TV-min), Wavelet soft-thresholding and Wiener filtering. For the TV-min algorithm we use the TVAL3 package provided by reference $^{18}$ whereas for the Wavelet and Wiener filtering we use the inbuilt MATLAB-2020a functions wdencmp and wiener2 respectively. The MATLAB code can be downloaded from the data availability statement.

Some example 2D images before and after filtering are shown in figure $\mathrm{S} 17$ for both step (top row) and continuous (bottom row) modes of the ODU. Note the 


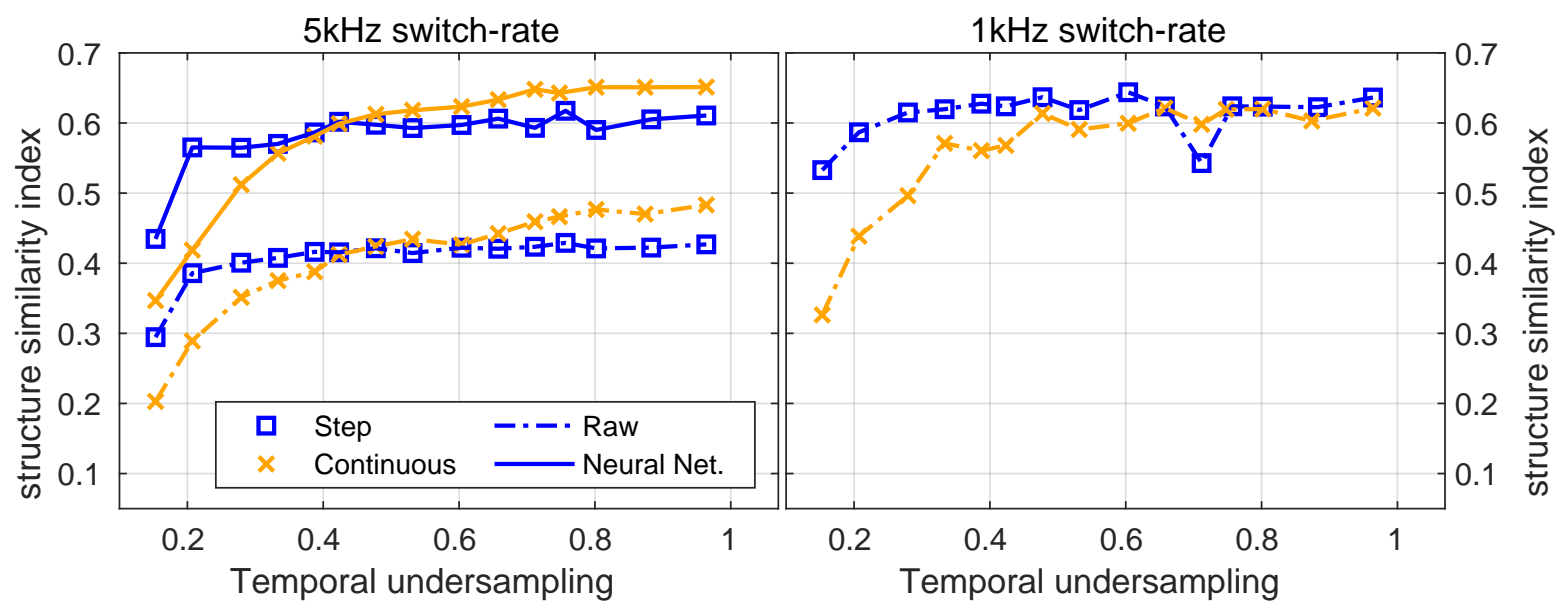

Figure S16. SSIM value of our 3D images as a function of temporal undersampling, with the left (right) handside graph being taken when the SLM switch-rate was 5 (1) $\mathrm{kHz}$.

step and continuous mode 2D-images are taken at different temporal points hence their differences. Figures S17a and $\mathbf{S 1 7 g}$ show the ground truth images obtained from averaging at a $1 \mathrm{KHz}$ switch-rate. Parts $\mathbf{b}$ and $\mathbf{h}$ are the raw images after post-processing the raw data as described in the previous supplementary sections. In parts $\mathbf{c}-\mathbf{f}$ and $\mathbf{i}-\mathbf{I}$ are the images after denoising by Wavelet soft-thresholding, TV-min, Wiener and our neural network respectively. It can be seen that all the techniques significantly improve the image quality. Comparing the processed images in figures S17c-f, we can see that the different denoising algorithms preserve different features whilst removing the noise. To evaluate the performance on these denoising techniques we calculate the SSIM of our 3D images as a function of temporal undersampling, which is shown in figure S18. It can be seen that all the algorithms produce the same trend with temporal undersampling but their absolute values are different, with the neural network having the highest SSIM followed by Wiener then Wavelet and finally TVmin. The reason why the neural networks performs the best can be seen by looking closely at figures S17h-I. Part $\mathbf{h}$ is the raw image and we can see some diagonal lines that are artefacts and they arise from a noise spike in one of the Fourier masks during our measurement. Small traces of these diagonal lines can be seen in all the denoised images however in the neural network they are nearly non-existent. Further, the neural network is the only one that has two peaks in top part of the image like the ground truth whereas the others only have a single peak. Although, sometimes the features can get blurred as can be seen by comparing the sharp line below the middle dark region in parts $\mathbf{a}$ and $\mathbf{f}$. However, the wavelet and Wiener filters appear to have better energy preservation by looking at the amplitudes of all the images.

Improving the image quality via denoising should be quick and not prevent the real-time display of the data. In regards to calculations times per single 2D image, TV-min took $18 \mathrm{~ms}$, wavelet took $3 \mathrm{~ms}$, the neural network took $0.15 \mathrm{~ms}$ and Wiener took about $0.13 \mathrm{~ms}$. This means that TV-min, wavelet, neural network and Wiener filtering can be used to process about 50, 320, 6500 and 7600 2D-image per second respectively. Taking into account the time needed for the processing needed in section S4, we find that TV-min and wavelet denoising are unable to display the images in real-time, ie. the total processing time becomes longer than the acquisition time, whereas the neural network (after training) and Wiener filtering can. These two techniques also give the highest SSIM values hence our work indicates that either of these two methods should be used. Wiener filtering is easier to setup whilst the neural network gives a better result at the expense of needing a lot of training data. 

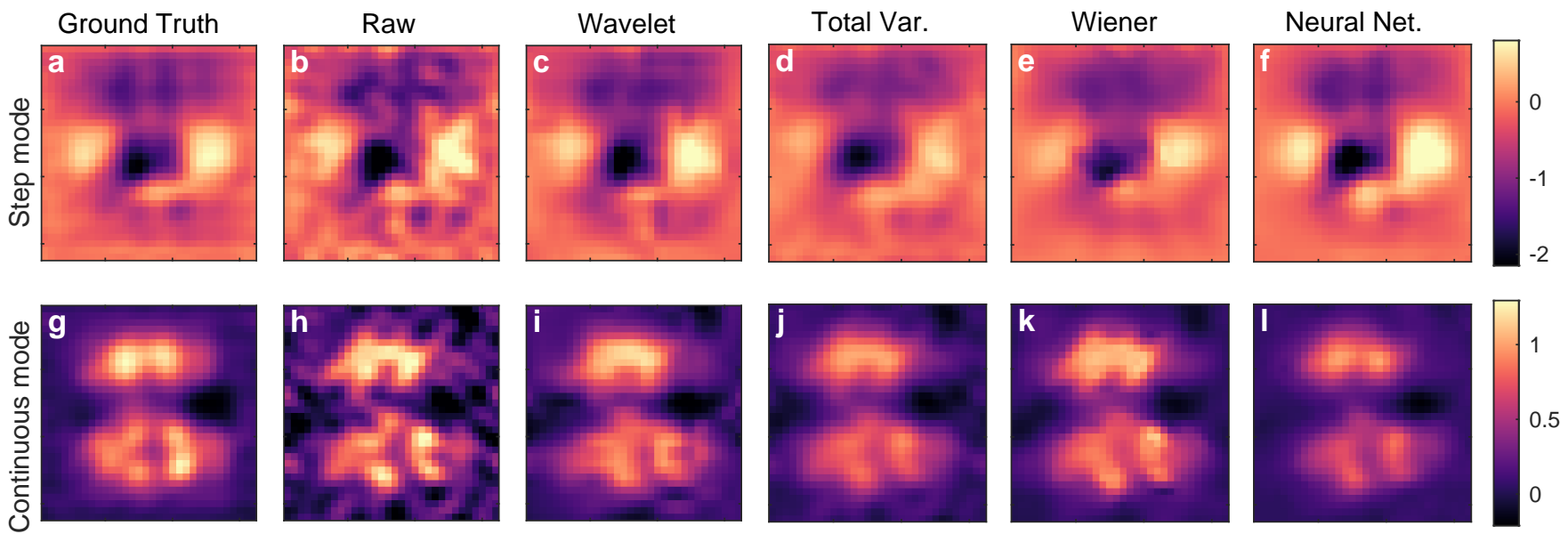

Figure S17. Raw and denoised 2D-images at single temporal point with a-f showing the ground truth, raw, wavelet denoised, total variational minimisation, Wiener filtering and image output from the neural network respectively. The ODU was in step mode in a-f and for parts $\mathbf{g}-\mathbf{I}$ it was in continuous mode.
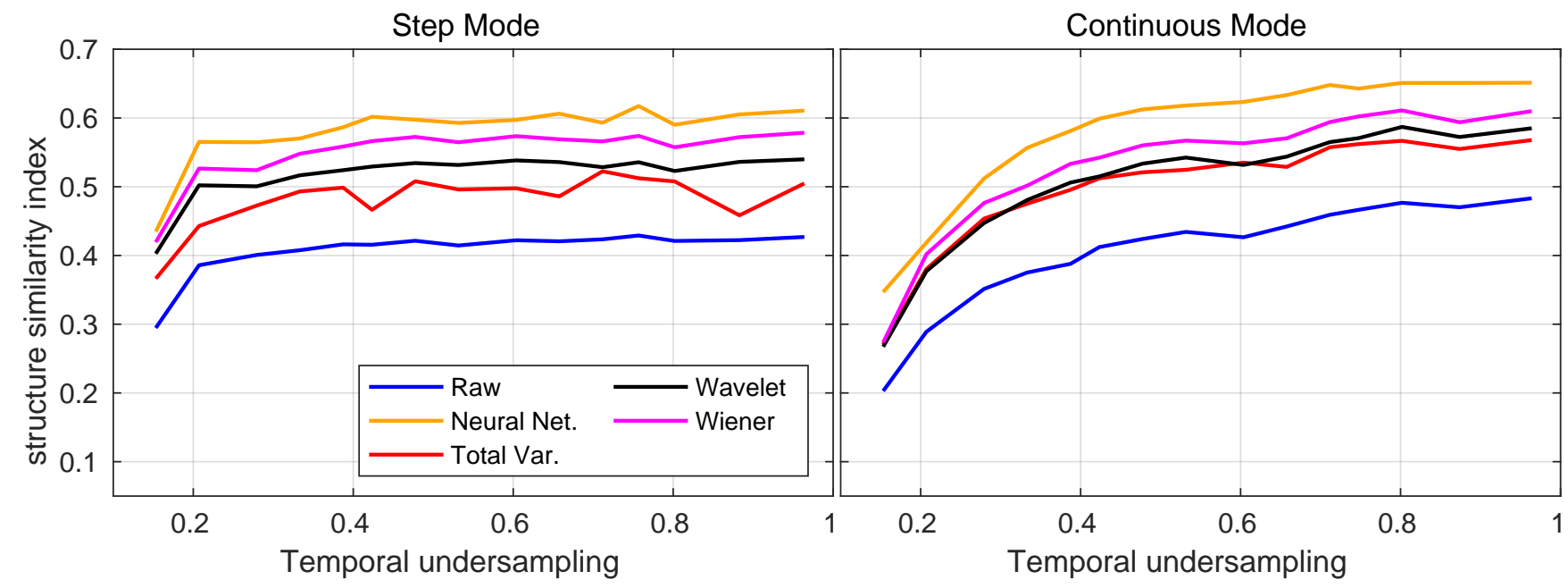

Figure S18. SSIM value of our 3D images as a function of temporal undersampling, with the left (right) handside graph being taken when the ODU was operating in step (continuous) mode.

\section{References}

[1] Stantchev, R. I., Yu, X., Blu, T. \& Pickwellmacpherson, E. Real-time terahertz imaging with a single-pixel detector. Nat. Commun. 11, 1-8, DOI: $10.1038 / \mathrm{s} 41467-020-16370-\mathrm{x}$ (2020).

[2] He, Y., Parrott, E. P. J. \& Pickwell-MacPherson, E. Adaptive Sampling for Terahertz Time-Domain Spectroscopy and Imaging. IEEE Transactions on Terahertz Sci. Technol. 7, 118-123, DOI: 10.1109/ TTHZ.2016.2640663 (2017).

[3] Pratt, W. K., Andrews, H. C. \& Kane, J. Hadamard Transform Image Coding. Proc. IEEE 57, 58-68, DOI: 10.1109/PROC.1969.6869 (1969).

[4] Decker, J. A. Hadamard-Transform Image Scanning. Appl. Opt. 9, 1392-1395, DOI: 10.1364/AO.
9.001392 (1970).

[5] Gourlay, J., McOwan, P., Vass, D. G., Underwood, I. \& Worboys, M. Time-multiplexed optical Hadamard image transforms with ferroelectricliquid-crystal-over-silicon spatial light modulators. Opt. Lett. 18, 1745-1747, DOI: 10.1364/OL.18. 001745 (1993).

[6] Watts, C. M. et al. Terahertz compressive imaging with metamaterial spatial light modulators. Nat. Photon. 8, 605-609, DOI: 10.1038/nphoton.2014. 139 (2014).

[7] Clemente, P. et al. Compressive holography with a single-pixel detector. Opt. Lett. 38, 2524-2527, DOI: 10.1364/OL.38.002524 (2013).

[8] Durán, V., Clemente, P., Fernández-Alonso, M., 
Tajahuerce, E. \& Lancis, J. Single-pixel polarimetric imaging. Opt. Lett. 37, 824-826, DOI: 10.1364/OL.37.000824 (2012).

[9] Yuan, J. \& Guo, C. A Deep Learning Method for Detection of Dangerous Equipment. In 2018 Eighth International Conference on Information Science and Technology (ICIST), 159-164, DOI: 10.1109/ICIST.2018.8426165 (2018).

[10] Higham, C. F., Murray-Smith, R., Padgett, M. J. \& Edgar, M. P. Deep learning for real-time singlepixel video. Sci. Reports 8, 2369, DOI: 10.1038/ s41598-018-20521-y (2018).

[11] Cai, B., Xu, X., Jia, K., Qing, C. \& Tao, D. DehazeNet: An End-to-End System for Single Image Haze Removal. IEEE Transactions on Image Process. 25, 5187-5198, DOI: 10.1109/TIP.2016. 2598681 (2016).

[12] Shi, J. et al. Automatic evaluation of traumatic brain injury based on terahertz imaging with machine learning. Opt. Express 26, 6371-6381, DOI: 10.1364/OE.26.006371 (2018).

[13] Caramazza, P. et al. Neural network identification of people hidden from view with a single-pixel, single-photon detector. Sci. Reports 8, 11945, DOI: 10.1038/s41598-018-30390-0 (2018).

[14] Sinha, A., Lee, J., Li, S. \& Barbastathis, G. Lensless computational imaging through deep learning. Optica 4, 1117-1125, DOI: 10.1364/OPTICA. 4.001117 (2017).

[15] Glorot, X. \& Bengio, Y. Understanding the difficulty of training deep feedforward neural networks. In Teh, Y. W. \& Titterington, M. (eds.) Proceedings of the Thirteenth International Conference on Artificial Intelligence and Statistics, vol. 9 of Proceedings of Machine Learning Research, 249-256 (JMLR Workshop and Conference Proceedings, Chia Laguna Resort, Sardinia, Italy, 2010).

[16] loffe, S. \& Szegedy, C. Batch Normalization: Accelerating Deep Network Trainixng by Reducing Internal Covariate Shift. In Bach, F. \& Blei, D. (eds.) Proceedings of the 32nd International Conference on Machine Learning, vol. 37 of Proceedings of Machine Learning Research, 448-456 (PMLR, Lille, France, 2015).

[17] Zhou Wang, Bovik, A. C., Sheikh, H. R. \& Simoncelli, E. P. Image quality assessment: from error visibility to structural similarity. IEEE Transactions on Image Process. 13, 600-612, DOI: 10.1109/TIP.2003.819861 (2004).

[18] Li, C., Yin, W., Jiang, H. \& Zhang, Y. An efficient augmented Lagrangian method with applications to total variation minimization. Comput. Optim. Appl. DOI: 10.1007/s10589-013-9576-1 (2013). 